\title{
Implantable Cardiac Device Infections Prevalence: Diagnostic and Therapeutic Implications
}

\author{
Hend Yahia ${ }^{1 * \mathbb{D}}$, Abdo Alazab $^{2}$, Randa Aly², Sameh Elmaraghi ${ }^{3}$, Ashraf Andraos $^{2}$ \\ ${ }^{1}$ Department of Critical Care, Faculty of Medicine, Helwan University, Helwan, Egypt; ${ }^{2}$ Department of Critical Care, Faculty of \\ Medicine, Cairo University, Cairo, Egypt; ${ }^{3}$ Department of Critical Care, Faculty of Medicine, Beni-Suef University, Beni Suef, Egypt
}

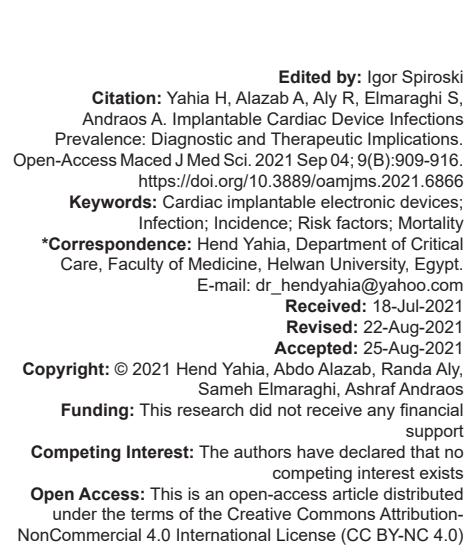

\section{Introduction}

In the 1960s, cardiac implanted electronic devices (CIEDs) were approved for routine clinical usage. Since then, their use has risen dramatically throughout the world, and they now contain cardiac resynchronization therapy (CRT) and implantable cardioverter defibrillators (ICDs) as well as permanent pacemakers. In numerous patient populations, CIEDs have been found to improve mortality and quality of life [1].

Implantation rates have risen over the previous many decades as a result of an aging population and an increasing number of individuals having arrhythmias, heart failure, or at a high rate of sudden cardiac death, all of which are associated with an increase in individual complexity and medical comorbidities [2].

CIEDs carry a potential for unfavorable events; classified as pulse generator complications (e.g. migration, erosion, and pocket infection $[\mathrm{PI}])$, procedurerelated complications (e.g., bleeding, pneumothorax, and perforation), and lead-related complications (e.g. displacement, fracture, and endocarditis) [3].
CIED-related infections are a serious complication having a rate of increase that exceeds the rate of new CIED implantation, with an approximate prevalence of $0.5-0.8 \%$ for primary implantation and $1-7 \%$ for secondary procedures [3].

Infections associated with CIEDs, whether localized PIs or systemic endovascular infections, have been linked to significant cost impact, mortality, and morbidity [4].

Numerous risk factors and comorbid disorders have been linked to CIED-related infection, including diabetes mellitus, underlying malignancies, operator inexperience, the patient's advanced age, previous anticoagulant or corticosteroid medication, and CIED placement in the recent history or secondary intervention, such as battery exchange [3].

Diagnosis of device infection accurately is necessary to ensure that individuals with infection receive proper treatment. Localized inflammation at the location of the pulse generator pocket is a certain sign of infection. Conversely, leads infection causes substantial diagnostic challenges, which can lead to delayed treatment administration and poor outcome [5]. 
Definitive diagnosis is also critical in patients who do not have a device infection to prevent unnecessary device removal since many patients are device dependent, and life-threatening consequences can arise from device removal [5].

In addition, infections that are related to CIEDs are difficult to be managed due to the presence of extracardiac and intracardiac constituents; both of these are susceptible to infection, and removing the device can be a big task with a potential of death or other consequences. As a result, prevention is critical [5].

\section{Study objectives}

The objectives of the study were to evaluate the prevalence of CIED-related infections, risk factors, clinical and demographic characteristics, causative organisms, and the management and outcome of patients presented in the Critical Care Department, Cairo University.

\section{Methods}

\section{Study population}

Our research study is a 10-year retrospective single-center observational non-controlled analysis of patients with evidence of CIED-related infections presented at the Critical Care Department of Cairo University Hospitals between January 2007 and December 2017.

The research study was conducted using the medical records of 1871 patients who had initial CIED implant or replacement during that period. Inclusion criteria were the patients who fulfilled the definition criteria for CIED-related infections according to the latest guidelines. We eliminated individuals with early post-implantation inflammation occurring within 30 days of implantation and manifesting only with erythema impacting the implantation incision site, without either purulent exudate, dehiscence, fluctuation, or systemic signs of infection [3].

Included patients were further segregated into two subgroups according to the site of infection:

1. Localized $\mathrm{PI}$, with local manifestations of infection with/without systemic manifestations. Positive blood culture or culture from the generator pocket provided more supportive evidence

2. CIED-related lead infection/endocarditis (CDE), with systemic manifestations of infection and echocardiographic detection of valvular or lead vegetation, or whether the modified major Duke criteria for infective endocarditis were met.

\section{Study design}

The following data were gathered from patients' medical records:

1. Demographic data

2. The time interval between the last manipulation of the device and the commencement of infection

3. CIED infection risk factors were classified as follows: (1) Patient-related risk factors; age, sex, immunosuppressive therapy, prior device infection; and the presence of renal failure, diabetes mellitus, chronic obstructive pulmonary disease (COPD), heart failure, malignancy, corticosteroid use, or anticoagulant use, (2) device-related risk factors; type and complexity of the used device and implantation of more than 1 lead, and (3) risk factors associated with the procedure; such as the absence of prophylactic antibiotics and reintervention procedures (including generator replacement, lead revision, and system upgrade)

4. Clinical evaluation including history and physical examination with special emphasis on vital signs, clinical manifestations of local infection (erythema, warmth, fluctuation, tenderness, wound dehiscence, erosion of the skin exposing the generator or leads, and purulent drainage), systemic manifestations of infection include fever, chills, night sweats, malaise, and anorexia

5. Device characteristics: Device types (ICD, $\mathrm{CRT}$, or pacemaker) and mode of pacing (VVI, DDD, CRTP, or CRTD)

6. Laboratory investigations including routine laboratories and microbiological studies

7. Transthoracic echocardiography (TTE); for the assessment of both the left and right ventricular dimensions and functions, signs of pulmonary hypertension, and presence of valvular and/ or lead vegetation and transesophageal echocardiography (TEE); to precisely measure location, structure, and size of vegetation. Vegetation was described as an oscillatory mass on cardiac valve leaflets, endocardial surface, or on the leads in the presence of a valve or lead infection, which was established by scanning in several echocardiographic views 8. Management and outcome.

\section{Statistical analysis}

The qualitative variables were expressed in terms of numbers and percentages, while the quantitative data were expressed in terms of variances, mean, and standard deviations. When the predicted count in any cell was $<5$, the Chi-square or Fisher's exact test was used to compare the groups. An independent t-test was used to compare two groups of individuals with reference to quantitative data with a parametric distribution. 
The association between two qualitative variables was determined using logistic regression analysis, along with its odds ratio and 95\% confidence interval $(\mathrm{Cl})$. The $95 \% \mathrm{Cl}$ was used, and a $5 \%$ margin of error was permitted. A statistically significant $p<0.05$ was considered.

\section{Results}

\section{Incidence}

A total of 2270 CIED procedures were performed for 1871 patients, and the total number of devices used was 1968. The implanted devices were 1591 permanent pacemakers (80.84\%), 324 CRT devices (16.47\%), and 53 ICD devices (2.7\%) (Table 1).

Table 1: Total number of implanted devices

\begin{tabular}{lll}
\hline Implanted device & Number & Percentage \\
\hline Single-chamber pacemakers & 483 & 24.54 \\
Dual-chamber pacemakers & 1108 & 56.30 \\
CRTP devices & 266 & 13.52 \\
CRTD devices & 58 & 2.95 \\
Single-chamber ICD devices & 21 & 1.07 \\
Dual-chamber ICD devices & 32 & 1.63 \\
Total devices & 1968 & 100 \\
\hline
\end{tabular}

ICDs: Implantable cardioverter defibrillators.

The number of other procedures (e.g. battery replacement, lead replacement, lead reposition, or new lead insertion) was 302.

De novo implantations represented $82.42 \%$ $(1871 / 2270)$ of total procedures, while recurrent procedures represented $17.58 \%$ (399/2270).

CIED infections were identified in 56 patients; two of them had recurrent infections, yielding 59 infectious episodes. The incidence of infectious episodes was $2.99 \%$ of totally inserted 1968 devices and $2.6 \%$ of total 2270 procedures.

The mean age of patients at the time of diagnosis was $60 \pm 18$ years (range 19-88). The male/ female ratio of infectious episodes was $27 / 32$.

The average time interval between the last device intervention and diagnosis was $2.25 \pm 2$ years (range 1 day-7 years).

The total number of CIED infections following de novo implantation was $22(37.29 \%)$, and those following recurrent procedures were 37 (62.71\%), representing $1.18 \%$ and $9.27 \%$ of total interventions, respectively $(p<0.001)$ (Table 2$)$.

Table 2: Percentage of CIED infection following de novo implantation and recurrent procedures in relation to total intervention number

\begin{tabular}{llllll}
\hline Procedure & $\begin{array}{l}\text { Infected } \\
\text { devices }\end{array}$ & $\begin{array}{l}\text { Total } \\
\text { intervention }\end{array}$ & Percentage & p-value & Sig. \\
\hline De novo implantation & 22 & 1871 & 1.18 & $<0.001$ & HS \\
Recurrent procedures & 37 & 399 & 9.27 & & \\
\hline CIED: Cardiac implanted electronic device. & & & &
\end{tabular}

Of all infectious episodes, 35 (59.32\%) patients had $\mathrm{PI}$, and 24 (40.68\%) patients had CDE, representing $1.54 \%$ and $1.06 \%$ of total procedures, respectively (Table 3 ).

Table 3: Cases with PI and CDE

\begin{tabular}{llll}
\hline $\begin{array}{l}\text { Site of } \\
\text { infection }\end{array}$ & Number & Percentage & $\begin{array}{l}\text { Percentage in relation to } \\
\text { total procedures }(n=2270)\end{array}$ \\
\hline $\mathrm{PI}$ & 35 & 59.32 & 1.54 \\
$\mathrm{CDE}$ & 24 & 40.68 & 1.06 \\
\hline CDE: CIED-related endocarditis. & &
\end{tabular}

\section{Devices characteristics}

All identified infected CIEDs were placed in the thoracic region with transvenous leads; 36 dualchamber pacemakers, 10 single-chamber pacemakers, seven CRTP devices, four CRTD devices, and two dual-chamber ICD devices. Patients with dual-chamber ICD and CRTD had the highest infection rate regarding totally implanted devices (Table 4).

Table 4: Percentage of infected devices regarding total devices number

\begin{tabular}{llll}
\hline Infected devices & Number & Total devices number & Percentage \\
\hline Pacemakers & 46 & 1591 & 2.89 \\
Single chamber & 10 & 483 & 2.07 \\
Dual chamber & 36 & 1108 & 3.25 \\
CRT devices & 11 & 324 & 3.4 \\
CRTP & 7 & 266 & 2.63 \\
CRTD & 4 & 58 & 6.89 \\
ICD devices & 2 & 53 & 3.77 \\
Single-chamber ICD & 0 & 21 & 0 \\
Dual-chamber ICD & 2 & 32 & 6.25 \\
\hline CRT: Cardiac resynchronization therapy, ICD: Implantable cardioverter defibrillator.
\end{tabular}

Infected devices with more than 1 lead represented $3.35 \%$ of totally inserted devices, yet; with no statistically significant difference from devices with one lead which represented $1.98 \%$ of totally inserted devices $(p=0.122)$.

\section{Risk factors \\ Patient related}

Diabetes mellitus was the most common risk factor in patients with CIED infection (30.51\%), followed by end-stage renal disease $(6.78 \%)$, previous device infection $(5.08 \%)$, anticoagulation (5.08\%), and COPD (3.39\%), respectively.

\section{Procedure related}

Reintervention was associated with a greater infection rate $(62.71 \%)$ followed by a lack of antibiotic prophylaxis $(6.78 \%)$.

\section{Device related}

Infection rates were significantly greater in patients with dual-chamber ICDs and CRTDs (Table 4), as well as in patients with more than 1 lead (83.05\%).

Comparing patients with $\mathrm{PI}$ with patients with CDE regarding risk factors, only reintervention procedures carried a highly significant statistical difference between both groups (48.57\% vs. $83.33 \%, p=0.007)$. 


\section{Clinical features}

Local manifestations of infection were skin erosion, purulent discharge, wound dehiscence, tenderness, fluctuation, hematoma, and erythema, while systemic manifestations of infection were fever, chills, and shock. Among patients with PI, the most common manifestation was skin erosion $(54.29 \%)$, while in patients with $\mathrm{CDE}$, fever was the most common presenting symptom $(62.5 \%)$.

\section{Microbiologic characteristics}

Blood samples and extracted lead cultures revealed bacterial growth in 26 cases $(44.07 \%$ ); bacterial growth was more in the group of CDE than the group of $\mathrm{PI}(66.67 \%$ vs. $28.57 \%, p=0.004)$. Grampositive bacteria represented $69.23 \%$ of the positive cultures, where staphylococci were isolated in $53.85 \%$ followed by both streptococci $(7.69 \%)$ and enterococci (7.69\%). Gram-negative bacteria represented $30.77 \%$ of all positive cultures (Figure 1).

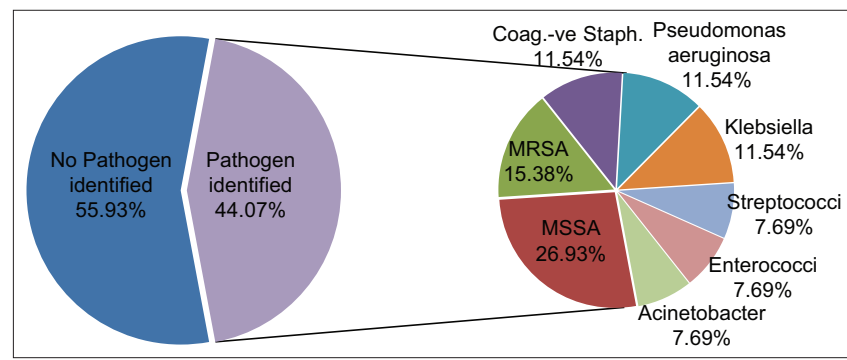

Figure 1: Blood and leads cultures

\section{Echocardiographic findings}

All cases had undergone TTE, and only 43 patients had undergone TEE. Leads vegetation was observed in 22 individuals and valvular vegetation in two individuals. The TEE offered additional diagnostic information for the identification of vegetation in seven cases.

\section{Management and outcome}

The average hospitalization time was 20 days (range 7-63 days). All patients received antimicrobial treatment with duration of antibiotics ranged between 1 and 6 weeks. In 53 cases, device removal has been carried out. Complete system extraction, including such generator and all accompanying leads, was accomplished in 41 cases and generator extraction alone took place in 12 cases. System extraction was percutaneously done in 29 patients and surgically by cardiothoracic surgeons through sternotomy in 12 patients. After a median period of 7 days, 49 new devices have been placed; 38 endocardial and 11 epicardial. Four patients did not have new devices installed (three of them had temporary pacemakers and died before insertion of the new permanent device while one case regained its own accelerated rhythm). The in-hospital mortality rate was $10.17 \%$ (six patients); three with multiorgan failure, two with septic shock, and one with pneumonia complicated with ARDS. The group of CDE had a greater mortality rate in comparison with the group of $\mathrm{PI}(20.83 \%$ vs. $2.86 \%, p=0.025)$.

\section{Discussion}

There has been a rise with the use of CIEDs in recent decades. Voigt et al. discovered that CIEDrelated infections were the major cause of death [6]. The incidence of infections associated with CIEDs has been shown to range between $0.5 \%$ and $2.2 \%$ [3], with an estimated infection rate of $0.5-0.8 \%$ following primary implant and $1-7 \%$ with secondary intervention [7], [8].

In the current study, it has been noted that the total number of individuals and implants was increased in comparison to a previous study that was done by the same department which studied 2367 patients having 2630 implanted devices over 25 years from 1982 till 2007 [9]. A total of 59 infectious episodes were identified with an incidence of $2.99 \%$ of totally implanted devices and $2.6 \%$ of total procedures, and this denotes that the incidence of infection was declining in comparison to old studies done by the same department, which varied between $3.6 \%$ and $4.4 \%$, respectively [9], [10], [11], [12].

In studies done by other centers, the results had varied; in a multicenter study done by Aydin et al., the infection prevalence was 1.6\% [13]. Besides, Kumar et al. indicated an overall infection incidence of $4.69 \% / 1000$ device years [14]. Moreover, Sadeghi et al. indicated an infection rate of $2.27 \%$ at 2.25 years [15]. In addition, Polyzos et al. showed an overall infection rate of $1.2 \%(0.3 \% \min -4.5 \% \max )$ for the 30 retrospectively cohorts included within their systematic review and meta-analysis of predisposing risk factors for CIED infection [16].

Numerous studies have found a variety of possible risk factors for CIED infection. Nonetheless, risk factors that were significant through one study were not significant in another and conversely. Patientrelated, procedure-related, and device-related factors have been identified. In our study, DM was associated with the highest potential of infection among the patientrelated risk factors. The incidence of diabetes in the CDE group was almost double that in the group of $\mathrm{PI}$, yet; it was not statistically significant $(41.67 \%$ vs. $22.86 \%$; $\mathrm{P}=0.123)$. The risk of infection in diabetic patients may be a consequence of the chronic hyperglycemia immunosuppressive impact. This finding is similar to the previous study done by the same department, 
which shows no statistically significant difference in the frequency of diabetes mellitus between the $\mathrm{PI}$ and endocarditis groups [9]. Similar results have been reported in other studies stating the high prevalence of diabetes in cases with CIED-related infection [15], [16], [17], [18]. On the other hand, Greenspon et al. found that individuals with diabetes had a decreased rate of CIED infection [19], and Klug et al. characterized diabetes as a negligible risk factor [20].

Regarding procedure-related risk factors, the average number of procedures performed before the presentation with infection was $1.85 \pm 0.8$. The infection rate was considerably greater in individuals who had repetitive procedures than in individuals who had only one procedure $(9.27 \%$ vs. $1.18 \%$; $p<0.001)$. Our findings supported other studies done by Kumar et al., Sadeghi et al., and Ludwigs et al. who showed that the infection rate was higher for recurrent procedures rather than de novo implants [14], [15], [21].

It is well established that recurrent procedures are one of the main risk factors of infection of the implanted prosthesis or device. It has been suggested that the increased rate of infection could result from diminished immunologic defenses within the constructed pocket and insufficient visibility of the surgical field [22]. In addition, it could be susceptible to bacterial contamination of the avascular capsule around the generator, impeding the passage of systemic antibiotics and inflammatory cells to the device pocket location [23], [24].

Amongst device-related risk factors, patients with dual-chamber ICD and CRTD had the highest infection rate regarding totally implanted devices, with an infection rate of $6.25 \%$ and $6.85 \%$, respectively. This correlates with other studies where it has been stated that the rate of infection is increased with more complex devices [15], [25], [26]. However, it contradicts a research study done by Kumar et al. who demonstrated that the infection rate did not increase in the group of patients with more complex devices [14].

The higher infection rate associated with complex devices is mainly correlated with an extended procedure duration, which has been associated with a higher risk of CIED-related infection. In addition, ICDs and CRTDs infection is most likely connected to the existence of many leads and a higher prevalence of concomitant comorbidities. The patient may also be predisposed to skin necrosis and consequent PI due to the large generator size [27].

Our study established that the existence of more than 1 lead is an important device-related risk factor as there was a higher infection rate in the patients who had devices with $>1$ lead than in those with only one lead ( $3.35 \%$ vs. $1.98 \%, p=0.122)$, and it was an independent risk factor in both groups of $\mathrm{PI}$ and CDE but with a higher significance in patients with CDE (74.29\% vs. $95.83 \%, p=0.03)$. Multiple pacing wires have been suggested as a possible cause of central venous thrombosis (in the vicinity of the leads), which may enhance the risk of device infection by acting as a nidus for subsequent seeding of pathogens [28].

Prior researches had established that bacterial infection is the primary cause of CIED infection, predominantly from normal skin flora, with Staphylococcus aureus and coagulase-negative staphylococci accounting for the majority of infections, accounting for $65-75 \%$ of generator Pls and up to $89 \%$ of CDE [29], [30]. In our series, bacterial growth was detected in $44.07 \%$, and it was significantly higher in the CDE group than in the PI group $(66.67 \%$ vs. $28.57 \%, p=0.004)$. Gram-positive bacteria were the most frequent causative organisms (69.23\%). Staphylococci were the most frequently isolated Gram-positive organisms in all cases (53.85\%) with an incidence in both groups of PI and CDE of $30 \%$ and $68.75 \%$, respectively.

Prior research on endocarditis caused by CIED has established the advantage of TEE over TTE in identifying vegetation [31], [32]. This was established in the current study; TEE offered additional diagnostic information in seven cases out of 43 patients who had undergone both TTE and TEE.

The optimal management of CIED infections requires device removal, especially in situations of lead endocarditis [3]. In our study, all cases received empirical broad-spectrum antibiotics with good coverage of staphylococcal infection and then antibiotics were modified according to culture results. Device removal was conducted in 53 cases (89.83\%); complete system removal (including the generator and all correlated leads) was done in 41 cases $(77.36 \%$ of removed devices), and removal of the generator only was done in 12 cases (all of them were in the group of $\mathrm{PI}$ ). Cases in the group of PI who had complete system removal; it was done through transcutaneous approach, while in the group of CDE, complete system removal was done in all cases; through transcutaneous approach in 10 cases and surgical approach in 12 cases $(45.45 \%$ and $54.55 \%$, respectively).

The mortality rate accompanying CIED infection is considerable and seems to be higher in patients with $\mathrm{CDE}$ than those with $\mathrm{PI}$. The overall reported infectionrelated mortality was ranging from $0 \%$ to $15 \%$ [3], while studies included only patients with CDE reported high mortality rate ranging from $24.5 \%$ to $29 \%$ [33], [34]. In our study, the mortality rate following CIED-related infection was found to be $10.17 \%$ (six patients), having a considerably higher prevalence in CDE individuals than in $\mathrm{PI}$ individuals $(20.83 \%$ vs. $2.86 \%$; $p=0.025)$.

\section{Limitations}

A retrospective study usually has the potential for referral bias and missing information such as 
recording periprocedural patient medical status, the precise interval between the start of symptoms and infection diagnosis, and follow-up records after recovery from an infectious episode. Besides, we cannot exclude the possibility that misclassification would undoubtedly alter our study findings. The prevalence and number of cases can be underrated due to a referral bias because our institution is a tertiary reference unit, and probably some less serious cases were handled locally without reaching us. Generator sizes, leads diameters, and leads materials have varied among different pacemaker devices that rely on the manufacturer and year of accessibility. This difference in the surface area of the generator and lead material may have an effect on bacterial adhesion features and the host's inflammatory response to the device and hence the possibility of CIED infection.

\section{Conclusion}

In our center, while CIED implants' rate continues to increase, the incidence rate of CIEDrelated infection is decreasing compared to earlier researches conducted by the same department. Yet, the infection burden associated with secondary intervention is still significantly high. The cumulative infection risk was significantly larger in individuals with diabetes mellitus, patients who had repeated procedures, and those with more complex devices or having devices with more than 1 lead. Blood culture and transesophageal echocardiography were the most efficient diagnostic tools and aided in the classification of patients. In the majority of instances with infection, particularly those with $\mathrm{CDE}$, the entire system removal was the management of choice. However, the mortality rate is still high.

\section{Recommendations}

CIED-related infection is an important and serious complication that better be avoided; so we recommend the following:

- Establishing a standardized checklist for appropriate interventional measures for modifiable risk factors; these measures include infection control measures, meticulous and strict aseptic/antiseptic techniques during the procedure, pre-procedural antibiotic prophylaxis, and homeostasis

- Comorbidity management should be a fundamental element of patient preparation, particularly before elective CIED treatments
Consideration of antimicrobial envelope, leadless pacemakers, and subcutaneous ICDs if feasible

Emphasis should be placed on patient education regarding recognizing infectionrelated symptoms and signs and the importance of regular follow-up with health-care providers Patients with CIED presented with any infective symptoms should be highly suspected and thoroughly investigated for CIED-related infection, especially those with identified risk factors

Regular surveillance of the rate of infection and providing the feedback to appropriate professionals to promote continual enhancement through clinical practice adjustment.

\section{References}

1. Epstein AE, DiMarco JP, Ellenbogen KA, Estes NM, Freedman RA, Gettes LS, et al. ACC/AHA/HRS 2008 guidelines for device-based therapy of cardiac rhythm abnormalities: A report of the American college of cardiology/American heart association task force on practice guidelines (writing committee to revise the ACC/AHA/NASPE 2002 guideline update for implantation of cardiac pacemakers and antiarrhythmia devices) developed in collaboration with the American association for thoracic surgery and society of thoracic surgeons. J Am Coll Cardiol. 2008;51(21):e1-62. https://doi.org/10.1161/ circualtionaha.108.189741

PMid:18498951

2. Uslan DZ, Tleyjeh IM, Baddour LM, Friedman PA, Jenkins SM St Sauver JL, et al. Temporal trends in permanent pacemaker implantation: A population-based study. Am Heart J. 2008;155(5):896-903. https://doi.org/10.1016/j.ahj.2007.12.022 PMid:18440339

3. Sandoe JA, Barlow G, Chambers JB, Gammage M, Guleri A, Howard $P$, et al. Guidelines for the diagnosis, prevention and management of implantable cardiac electronic device infection. Report of a joint working party project on behalf of the British society for antimicrobial chemotherapy (BSAC, host organization), British heart rhythm society (BHRS), British cardiovascular society (BCS), British heart valve society (BHVS) and British society for echocardiography (BSE). J Antimicrob Chemother. 2015;70(2):325-59. https://doi.org/10.1093/jac/ dku383

PMid:25355810

4. Reynolds MR, Cohen DJ, Kugelmass AD, Brown PP, Becker ER, Culler SD, et al. The frequency and incremental cost of major complications among medicare beneficiaries receiving implantable cardioverter-defibrillators. J Am Coll Cardiol. 2006;47(12):2493-7. https://doi.org/10.1016/j.jacc.2006.02.049 PMid:16781379

5. İpek EG, Guray U, Demirkan B, Guray Y, Aksu T. Infections of implantable cardiac rhythm devices: Predisposing factors and outcome. Acta Cardiol. 2012;67(3):303-10. https://doi. org/10.1080/ac.67.3.2160719

PMid:22870738

6. Voigt A, Shalaby A, Saba S. Continued rise in rates of 
cardiovascular implantable electronic device infections in the United States: Temporal trends and causative insights. Pacing Clin Electrophysiol. 2010;33(4):414-9. https://doi. org/10.1111/j.1540-8159.2009.02569.x

PMid:19793359

7. Baddour LM, Epstein AE, Erickson CC, Knight BP, Levison ME, Lockhart PB, et al. Update on cardiovascular implantable electronic device infections and their management: A scientific statement from the American Heart Association. Circulation. 2010;121(3):458-77. https://doi.org/10.1161/ circulationaha.109.192665

PMid:20048212

8. NeryPB,FernandesR, NairGM,SumnerGL, RibasCS,MenonSM, et al. Device-related infection among patients with pacemakers and implantable defibrillators: Incidence, risk factors, and consequences. J Cardiovasc Electrophysiol. 2010;21(7):78690. https://doi.org/10.1111/j.1540-8167.2009.01690.x

PMid:20102431

9. Andraos AW, Hussein K, Zaky SH, Hamed G, Omar SH, Aly R, et al. Prevalence and outcome of bacterial endocarditis in patients with implantable cardiac devices infections. Saudi Heart Assoc. 2009;21(2):100-6.

10. Tayeh OM, Gharib S, Mokhtar S. Short Versus Long Term Follow up of Permanent Pacemakers. Ten Years Experience at Cairo University, Doctoral Dissertation, MSc Thesis, Cairo University; 1995.

11. Moharam A, Radwan W, Mokhtar S. Long Term Evaluation of Implanted Cardiac Pacemakers. A Fifteen Years Registry of the Critical Care Medicine Department, Doctoral Dissertation, MSc Thesis, Cairo University; 2000.

12. Andraos AW, Zaky SH, Abdo A. Pocket infection and pacemaker lead endocarditis, pacemaker lead infective endocarditis: Is it related only to pocket infection. N Egypt J Med. 2003;29:LI. https://doi.org/10.1016/s1099-5129(03)91727-2

13. Aydin M, Yildiz A, Kaya Z, Kaya Z, Basarir AO, Cakmak N, et al. Clinical characteristics and outcome of cardiovascular implantable electronic device infections in Turkey. Clin Appl Thromb Hemost. 2016;22(5):459-64. https://doi. org/10.1177/1076029614567310 PMid:25589093

14. Kumar A, Kumar A, Goel PK. Cardiac device related infection: A study from a tertiary care hospital in India. Ann Int Med Dent Res. 2017;3(4):4. https://doi.org/10.21276/aimdr.2017.3.4.me2

15. Sadeghi H, Alizadehdiz A, Fazelifar A, Emkanjoo Z, Haghjoo M. New insights into predictors of cardiac implantable electronic device infection. Tex Heart Inst J. 2018;45(3):128-35. https:// doi.org/10.14503/thij-17-6300 PMid:30072848

16. Polyzos KA, Konstantelias AA, Falagas ME. Risk factors for cardiac implantable electronic device infection: A systematic review and meta-analysis. Europace. 2015;17(5):767-77. https://doi.org/10.1093/europace/euv053 PMid:25926473

17. Hercé B, Nazeyrollas $P$, Lesaffre F, Sandras R, Chabert JP, Martin A, et al. Risk factors for infection of implantable cardiac devices: Data from a registry of 2496 patients. Europace. 2013;15(1):66-70. https://doi.org/10.1093/europace/eus284 PMid:23097224

18. Prutkin JM, Reynolds MR, Bao H, Curtis JP, Al-Khatib SM, Aggarwal S, et al. Rates of and factors associated with infection in 200909 Medicare implantable cardioverter-defibrillator implants: Results from the national cardiovascular data registry. Circulation. 2014;130(13):1037-43. https://doi.org/10.1161/ circulationaha.114.009081

PMid:25081281
19. Greenspon AJ, Patel JD, Lau E, Ochoa JA, Frisch DR, Ho RT, et al. 16-year trends in the infection burden for pacemakers and implantable cardioverter-defibrillators in the United States: 1993 to 2008. J Am Coll Cardiol. 2011;58(10):1001-6. https://doi. org/10.1016/j.jacc.2011.04.033

PMid:21867833

20. Klug D, Balde M, Pavin D, Hidden-Lucet F, Clementy J, Sadoul N, et al. Risk factors related to infections of implanted pacemakers and cardioverter-defibrillators: Results of a large prospective study. Circulation. 2007;116(12):1349-55. https:// doi.org/10.1161/circulationaha.106.678664

\section{PMid:17724263}

21. Ludwig S, Theis C, Brown B, Witthohn A, Lux W, Goette A Incidence and costs of cardiac device infections: Retrospective analysis using German health claims data. J Comp Eff Res. 2018;7(5):483-92. https://doi.org/10.2217/cer-2017-0080 PMid:29132224

22. Lekkerkerker JC, van Nieuwkoop C, Trines SA, van der Bom JG, Bernards A, van de Velde ET, et al. Risk factors and time delay associated with cardiac device infections: Leiden device registry. Heart. 2009;95(9):715-20. https://doi. org/10.1136/hrt.2008.151985

PMid: 19036758

23. Pichlmaier M, Marwitz V, Kühn C, Niehaus M, Klein G, Bara C et al. High prevalence of asymptomatic bacterial colonization of rhythm management devices. Europace. 2008;10(9):1067-72. https://doi.org/10.1093/europace/eun191 PMid: 18658253

24. Subbiahdoss G, Kuijer R, Grijpma DW, van der Mei HC Busscher HJ. Microbial biofilm growth vs. tissue integration: "The race for the surface" experimentally studied. Acta Biomater. 2009;5(5):1399-404. https://doi.org/10.1016/j. actbio.2008.12.011

PMid:19158003

25. Ann HW, Ahn JY, Jeon YD, Jung IY, Jeong SJ, Joung B, et al. Incidence of and risk factors for infectious complications in patients with cardiac device implantation. Int $\mathrm{J}$ Infect Dis. 2015;36:9-14. https://doi.org/10.1016/j.ijid.2015.05.011 PMid:25980618

26. Køber L, Thune JJ, Nielsen JC, Haarbo J, Videbæk L, Korup E, et al. Defibrillator implantation in patients with nonischemic systolic heart failure. N Engl J Med. 2016;375(13):1221-30. https://doi.org/10.1056/nejmoa1608029 PMid:27571011

27. Romeyer-Bouchard C, da Costa A, Dauphinot V, Messier M, Bisch L, Samuel B, et al. Prevalence and risk factors related to infections of cardiac resynchronization therapy devices. Eur Heart J. 2010;31(2):203-10. https://doi.org/10.1093/eurheartj/ ehp421

PMid:19875388

28. Howarth DM, Curteis PG, Gibson S. Infected cardiac pacemaker wires demonstrated by Tc-99m labeled white blood cell scintigraphy. Clin Nucl Med. 1998;23(2):74-6. https://doi. org/10.1097/00003072-199802000-00002 PMid:9481492

29. Bongiorni MG, Tascini C, Tagliaferri E, Cori AD, Soldati E, Leonildi $A$, et al. Microbiology of cardiac implantable electronic device infections. Europace. 2012;14(9):1334-9. https://doi. org/10.1093/europace/eus044 PMid:22399202

30. Sohail MR, Uslan DZ, Khan AH, Friedman PA, Hayes DL, Wilson WR, et al. Management and outcome of permanent pacemaker and implantable cardioverter-defibrillator infections. J Am Coll Cardiol. 2007;49(18):1851-9. https://doi.org/10.1016/j. jacc.2007.01.072 
PMid: 17481444

31. Victor F, de Place C, Camus C, Le Breton H, Leclercq C, Pavin D, et al. Pacemaker lead infection: Echocardiographic features, management, and outcome. Heart. 1999;81(1):82-7. https://doi.org/10.1136/hrt.81.1.82

PMid:10220550

32. Klug D, Wallet F, Lacroix D, Marquie C, Kouakam C, Kacet S, et al. Local symptoms at the site of pacemaker implantation indicate latent systemic infection. Heart. 2004;90(8):882-6. https://doi.org/10.1136/hrt.2003.010595
PMid:15253959

33. Athan E, Chu VH, Tattevin P, Selton-Suty C, Jones $P$, Naber $\mathrm{C}$, et al. Clinical characteristics and outcome of infective endocarditis involving implantable cardiac devices. JAMA. 2012;307(16):1727-35. https://doi.org/10.1001/jama.2012.497 PMid:22535857

34. Baman TS, Gupta SK, Valle JA, Yamada E. Risk factors for mortality in patients with cardiac device-related infection. Circ Arrhythm Electrophysiol. 2009;2(2):129-34. https://doi. org/10.1161/circep.108.816868

PMid:19808457 